\title{
Contrastive Study of Hedges in English-Chinese Social Science Articles
}

\author{
Lili Sun \\ College English Teaching Department \\ Zao Zhuang University \\ Zao Zhuang, China
}

\begin{abstract}
Through self-built English and Chinese corpora, this paper tries to compare the differences and similarities of the use of hedges. With the help of SPSS and AntConc software, we can get that there are obvious differences and similarities in different scales of the two corpora. The result can be analyzed from two aspects. One is from linguistic perspective and the other one is from cultural differences. The result of the study can provide reference for thesis writing standards to reduce the phenomenon of negative transfer of mother tongue in essay writing. Meanwhile, it makes us to have a better understanding between Chinese and western culture.
\end{abstract}

Keywords-hedges; English-Chinese corpora; scales; differences; similarities

\section{INTRODUCTION}

Hedges are widely used in various linguistic contexts. Looking through previous studies, hedges can be easily found in scientific discourse, legal discourse, diplomatic discourse, business articles and news articles. But the frequency of the use of hedges is different (Hinkel 1997). In recent years, the study of hedges is mainly found in scientific discourse. Hyland (1996) made research on functional features of hedges in scientific articles. The research of Hyland(1998), $\mathrm{Hu}$ and $\mathrm{Cao}(2011)$ are the most representative in the field of abstracts of scientific articles. In various genres of discourse, the use of hedges in written articles especially in scientific discourse has become the hot issue of researchers. Domestic linguistic scholars make it from communicative aspects combined with cooperative principles and politeness principles. Series of research are undertaken from its pragmatic function (Gao 2002). Some scholars study the use of hedges in natural science English articles; some study the frequency of hedges in abstracts of English scientific articles and those in applied linguistic articles (Li 2011, Zeng 2005). Through the search for the study of hedges in recent years, it can be inferred that qualitative study are much more than quantative studies. Contrastive study between English and Chinese or between any other languages can be hardly found.

\section{Classification AND FUnCtions OF HEDGES}

According to the American linguists Ellen Prince, hedges can be divided into two kinds, namely, approximators and shields from semantic perspective. Approximator refers that the speakers make fuzzy expressions on the propositional content. Shields refer to the fuzzy expression of speakers' attitude toward proposition. It refers to some model verbs expressing some possibility and some epistemic adjectives, adverbs and nouns. Hyland (1998) made the classification of hedges used in scientific articles. They are model verbs expressing possibility. Based on previous studies, this study will focus on the contrastive use of hedges in English and Chinese articles.

Lakoff(1972:471) gave the definition of hedges like this: Words whose job it is to make things more or less fuzzy. According to previous studies, in scientific discourse, hedges are widely used to express the hypothesis and deductions of research results. The use of hedges can lessen the certainty and the validity of the authors' statement. So it is widely used in scientific discourse.

Apart from the cognitive functions, Strauss (2004) mentioned that the use of hedges has another two functions. One is politeness, the other one is cultural factors. From the politeness perspective, it can avoid the offense of the scholars in related research fields. For cultural factors, the use of hedges can avoid the clash of different point of view in the same research field. Namely, the use of hedges can lessen or alleviate the interpersonal relationship in academic fields. In academic fields, scholars may hold argumentative views or contradictory views. The expressions of hedges can make the authors' point of view more accurate, more objective, and more serious.

In addition to the cognitive and communicative functions, Jensen (2008) found that the approximate use of hedges can add more incredibility of a scientific report. The limitation in scientific reports is the reflection of the incredibility, so hedges in the limitation parts can add more credibility in scientific research fields.

\section{RESEARCH AND METHOD}

\section{A. Research Questions}

Based on previous studies, this paper will make the word list of hedges more complete. With the self-built English and Chinese corpora, this paper will aim to prove the following hypothesis:

- To examine the overall frequency of hedges in the two corpora. 
- To find out the frequency and distribution of various forms of hedges in the corpora.

- To analyze the reason of the different attribution of hedges in both the Chinese and English articles.

\section{B. Corpus Sources and Methodology}

This paper is mainly based on two self-built corpora. One is Chinese and the other one is English. Each corpus consists of 10 academic articles related to social sciences. The authors of the ten English articles are native English speakers. And the ten Chinese articles are written by native Chinese speakers. All these paper are published during the year20012005. All the English and Chinese articles are selected from different four fields, namely, linguistics, anthropology, education and sociology.

The English articles are selected from the well-known international academic journals. They are Journal of Pragmatics, Contemporary Sociology, American Journal of Education, Current Anthropology. The Chinese articles are selected from the domestic famous journals. They are Journal of Educational Research, Journal of Sociology Study, Contemporary Linguistics, and Journal of Anthropology. In this paper, the Chinese corpus is called CJ, and the English corpus is called EJ.

In this paper, the classification of hedges is based on lexical and grammatical level. According to Swales theory on each sections, the author divide each articles into three sections, namely, introduction, method, result and discussion.

So how to identify each hedges? This paper studies the hedges according to the criterion of Ken Hyland(1998). During the study, the author counted from four aspects, that is, modal verb; lexical verbs; epistemic adjective, adverb and noun and other phraseological expression.

The analysis of hedges in Chinese and English are as follows:

\section{- Model verbs}

Example (1) ... 这将有助于他们...(meaning:...that will help them...)

Example (2) The adult reader/writer and the child reader will be exposed to ...

In example (1) and (2), the model verbs “将”and "will” express the prediction of what will happen and appear.

Example (3)南方族群可分为...(meaning: The southern ethnicity group can be divided into...)

Example (4) Only further research_can assess whether this is true...

In example (3) and (4), “可” and “can” express the 对某 事的肯定的预测.

- Lexical adjective, adverbs and nouns

Example (5) 此部分结果也间接支持了相互性孝道的重要 性相对高于权威性(Meaning: The result in this part also indirectly supports that the importance of filial piety is relatively higher than that of authority)

Example (6) ...保持了较平稳的发展...(meaning：...keep more steady developing state.)

Example (7)..., 在不同期次间皆存在显著差异

(meaning: There are indirect significant differences among periods )

Example (8) precisely because the selectivity and scope were relatively high at this school.

The expression in the above examples“相对”, “较”, “显 著" and "relatively" are used to lessen the certainty and make the expression more indirect.

- Lexical verbs

Example (9) 我们认为 $\cdots$, 它们作用不同。(meaning: We believe that..., they play different roles)

Example (10) ... over two-thirds believe that land occupations are the most effective way.

Example (11) We suggest that more research is needed on this issue.

The expressions “认为”, “believe”和 “suggest" can express the judgment of a certain person or a group of people on something.

- Phraseological expression

Example (12) ..., 受试普遍认为...(meaning: The subjects generally believe that...)

Example (13)... teaching practices in general education courses may predict or influence students.

In Example (12) and Example (13)，“普遍认为”, “in general" are used to summarize, not used to express personal views.

During the research, the software AntConc3.2.1 and SPSS19.0 is employed. With the assistance of the software AntConc3.2.1, the data are counted more quickly, accurately. With the software SPSS19.0, the data of the attribution of hedges can make the difference clearly.

\section{RESULTS AND ANALYSIS}

A. The statistic result

As "Table I" and "Table II" shown, there are some differences in English and Chinese corpora.

TABLE I. THE FREQUENCY OF HEDGES IN EACH CORPORA

\begin{tabular}{|c|l|l|l|}
\hline Corpus & $\begin{array}{c}\text { TotalNo. of } \\
\text { Words }\end{array}$ & \multicolumn{1}{|c|}{$\begin{array}{c}\text { Total No. of } \\
\text { Hedges }\end{array}$} & $\begin{array}{c}\text { Hedges Per } \\
\text { 1000Words }\end{array}$ \\
\hline $\boldsymbol{E J}$ & 59,456 & 1657 & $27.9 \%$ \\
\hline $\boldsymbol{C J}$ & 90,780 & 1917 & $21.1 \%$ \\
\hline
\end{tabular}

The data in "Table I" shows that the proportion of hedges in one thousand words is $27.9 \%$ in English corpus. However, in Chinese corpus the proportion of the hedges is $21.1 \%$. So the frequency of the hedges in EJ is obviously higher than that in CJ. The result is that Chinese scholars express their opinions more definitely and indirect express is rarely used. 
But for the native English speakers, they use more indefinite and indirect expression to lessen the certainty of their attitude. They are more cautious when they express their opinions.

In "Table II", we summarize the distribution of hedges in each section. It is shown that there are some differences and similarities. The common points are as follows. Firstly, both in EJ and CJ, the distribution of hedges are the highest in section 3 and the proportion is $76 \%$ 和 $63 \%$ respectively. Secondly, the frequency of hedges in both corpora is higher in section 1 . The result is $17 \%$ 和 $24 \%$ respectively. The frequency of hedges in section 2 is the lowest, namely, 7\% 和 $13 \%$ in EJ and CJ. In introduction section, the author mainly objectively states background information, so fuzzy statement is seldom used in this part. In method section, the author mainly introduces his research purpose, research object and method. All the statement is objective. The authors' point of view is not shown in this part. So the use of hedges is fewer.

TABLE II. DISTRIBUTION OF HEDGES IN VARIOUS SECTIONS

\begin{tabular}{|c|c|c|c|c|}
\hline $\begin{array}{c}\text { Corp } \\
\text { ora }\end{array}$ & $\begin{array}{c}\text { Introduct } \\
\text { ion 1 } \\
(\text { raw } \\
\text { no., \%) }\end{array}$ & $\begin{array}{c}\text { Method 2 } \\
\text { (raw } \\
\text { no., \%) }\end{array}$ & $\begin{array}{c}\text { Result\&Discussi } \\
\text { on 3 } \\
\text { (raw no., \%) }\end{array}$ & $\begin{array}{c}\text { Total } \\
\text { (raw } \\
\text { no., \%) }\end{array}$ \\
\hline $\boldsymbol{E J}$ & $281(17 \%)$ & $115(7 \%)$ & $1259(76 \%)$ & 1657 \\
\hline $\boldsymbol{C J}$ & $460(24 \%)$ & $257(13 \%)$ & $1200(63 \%)$ & 1917 \\
\hline
\end{tabular}

The distribution of hedges in results and discussion section is the highest. This part is the most important part for the author to analyze the research result and to compare with the previous studies. And the author will provide the suggestion and prediction of the research. The employment of hedges can help the author to state the research result and hypothesis cautiously. Meanwhile, it can make the research more objective and accurate. In section 3, the opinions and views of the author will be elaborated and the possibility and prediction will be inferred. On the one hand, the author should explain his own research result and findings clearly. On the other hand, the author should express his own opinions euphemistic. So the frequency of hedges in this part is the highest.

But there are still some differences of distribution in CJ and EJ. In CJ, the distribution of hedges in method section and introduction section is $13 \%$ and $24 \%$. The result is obviously higher than that of in EJ. But in EJ, the distribution of hedges in sections is $76 \%$, however, in CJ, it's only $63 \%$. For the various forms of hedges in each section of the two corpora, there are also some differences. As is shown in "Table III", the distribution of hedges in EJ is lexical adjectives, adverbs and nouns are the most, lexical verbs are more and phraseological expression is the least. In CJ, the distribution of various forms of hedges is similar to EJ.

In CJ, the distribution of phraseological expression is lower than that of in EJ. The frequency of epistemic adj., n. $\&$ adv. is the highest in CJ and that of model verbs is higher. The data shows that Chinese scholars are used to employing epistemic adj., n. \& adv. to alleviate their statements and they seldom use phraseological expression. The frequency of model verbs is higher in EJ than that of in CJ.

TABLE III. DISTRIBUTION OF VARIOUS FORMS OF HEDGES

\begin{tabular}{|c|c|l|l|l|}
\hline $\begin{array}{c}\text { corpo } \\
\text { ra }\end{array}$ & $\begin{array}{c}\text { Modal V. } \\
\text { (raw } \\
\text { no., \%) }\end{array}$ & $\begin{array}{c}\text { Lexical } \\
\text { V.(raw } \\
\text { no., \%) }\end{array}$ & $\begin{array}{c}\text { Epistemic } \\
\text { adj.,n.\&adv } \\
\text { (raw no., \%) }\end{array}$ & $\begin{array}{c}\text { phraseological } \\
\text { expression } \\
\text { (raw no., \%) }\end{array}$ \\
\hline $\boldsymbol{E J}$ & $486(29 \%)$ & $\begin{array}{l}171(10 \\
\%)\end{array}$ & $842(51 \%)$ & $158(10 \%)$ \\
\hline $\boldsymbol{C J}$ & $482(20 \%)$ & $\begin{array}{l}375(20 \\
\%)\end{array}$ & $1045(54 \%)$ & $15(6 \%)$ \\
\hline
\end{tabular}

\section{A. Discussion}

Comparing all the figures in the above tables, it is found that there are some similarities and differences in the use of hedges. By analyzing all the phenomena, we can explain the reasons from two aspects. One is language factors and the other one is social cultural differences.

1) Language factor-Chinese author seldom uses the variety of hedges.

As the data shown, the frequency of hedges in Chinese articles is obviously lower than that of in English articles. The research result is consistent to the findings that Hyland got in one of his papers in 1998. Through the data, we can also find that when Chinese writers express the fuzzy meaning, they seldom use the forms of hedges. Although some hedges can be found, the form of hedges is single, not so various. We can take some model verbs for example. In $\mathrm{CJ}$, when the author express the prediction to the future, he will employ the word “将” only, without any other forms. But in EJ, the words “will” 和 “would" are frequently used to express the prediction of the future. And there are some differences between the words "will" 和 "would". When they express the prediction, the degree of the tone is different. The word "will" expresses the possibility much stronger than the word "would". But in CJ, there is no difference of the degree of possibility. The word “将" is used consistently when the prediction of possibility is expressed.

TABLE IV. HEDGES OF NUMERICAL DATA IN EJ AND CJ

\begin{tabular}{|c|l|l|l|}
\hline 近似 & 3 & nearly & 7 \\
\hline 左右 & 3 & Slightly & 7 \\
\hline 几乎 & 3 & about & 9 \\
\hline 许多 & 7 & approximately & 13 \\
\hline 略 & 8 & many & 58 \\
\hline 约 & 9 & most & 70 \\
\hline 多数 & 10 & more & 83 \\
\hline 一些 & 14 & some & 94 \\
\hline 近 & 38 & less & 38 \\
\hline
\end{tabular}

Apart from differences of model verbs, there are some differences in data presentation. In EJ, the words "about, approximately, some, around" are often used to expression approximate number but in EJ, we only use “约”correspondingly. When we expression approximate number, we often use “几”, “许多”, “多”, “多数”, “左右”, “和”... “以上” etc “Table IV”. The forms are different when the Chinese and English writers express approximately. But by corpus, the amount of approximate number in EJ is much higher than that of in CJ. From this phenomenon, we can get that when English writer express their own opinion, 
they are more cautious and employ more approximate number to express their uncertainty, but for Chinese authors, things are opposite.

In both $\mathrm{CJ}$ and EJ, hedges used with numerical data are different. From the table, it is clear that there are more forms of hedges in EJ and the frequency of the hedges is much higher than that of in CJ. With the help of the software SPSS, the data is compared and the value is $0.022(<$ sig.0.05), so it is further proved that there is obvious differences between the two corpora.

\section{2) Cultural Factors}

By analyzing the different use of model verbs and hedges of data numerical, it is clear that the use of hedges is different in language expressions in academic articles. The use of hedges not only expresses the different language phenomenon, but reflects the rules and traditions hidden behind the phenomenon. So we can exp lain the differences from cultural perspective.

The American psychologist Fromm points that thinking pattern of Easterners and Westerners is different. Easterners tend to be synthetical, collective and direct, but westerners tend to be analytic, individual and objective. It can be embodied differently in writing. Easterners express their views directly and they think when a scientific argument is raised, it should be correct and accurate so hedges are not used frequently in their articles. And they hold the view that the use of hedges will lessen the credibility of their argument. In opposite, when western scholars express their opinions, they seldom express it too definitely and absolutely. Their analytic thinking pattern makes them undertake researching and explore to proceed. They are inclined to provide their hypothesis and then verify it. So the use of hedges is an important in their research process. And the use of hedges can enhance the incredibity.

\section{CONCLUSION AND LIMITATION}

This paper has found that there are some differences in using hedges for Chinese writers and English writers. Generally speaking, the proportion of frequency of hedges in each section in both CJ and EJ is similar. But the total amount of hedges is much higher in EJ than in CJ.

In EJ and CJ, the amount of hedges used in section 3 (result \& discussion) is the highest. The various forms of hedges in both corpora are different. In CJ, the most frequently used form is epistemic adjectives, nouns and adverbs. Phraseological expression is seldom used. In EJ, model verbs are used more frequently than that in CJ and different model verbs can express different meanings with different tones. But in $\mathrm{CJ}$, model verbs are used in single forms and the mode is not colorful.

Forms of hedges used with numerical data are fewer in CJ than that in EJ. It can be attributed to the different thinking patterns. Asian thinking pattern tends to be descriptive, synthetic and collective, but westerners tend to be analytic, individual. So Chinese people like to express their opinions directly and westerners tend to express their opinions evolutionary, not so directly.
This study tries to find the use of hedges in Chinese and English journals. The findings can provide some international criterion for paper writing. And it can provide some reference for Chinese writers to avoid the negative transfer in paper writing. Meanwhile, it provides a further study for eastern and western cultures. But the objects of this study are only articles related to social sciences, not including all fields of sciences. So its application is still limited.

\section{REFERENCES}

[1] Gao Xiaofang\&Zhang Qin. Hedges: categorization and application. Journal of Sichuan International Studies University, 2002(5):89-91.

[2] Hinkel, E. Indirectness in L1 and L2 academic writing. Journal of Pragmatics, 1997(27):361-365.

[3] Hu Guangwei \& Cao Feng. Hedging and boosting in abstracts of applied linguistics articles: a comparative study of English-and Chinese-medium Journals. Journal of Pragmatics, 2011(43):27952799.

[4] Hyland, K. Nurturing hedges in the ESP curriculum. System, 1996(4):1473-1492.

[5] Hyland, K. Hedging in Scientific Research Articles. John Benjamins Publishing, Amsterdam/Philladelphia, 1998.

[6] Jesen, J. Scientific uncertainly in News coverage of cancer research: effects of hedging on scientists' and journalists' credibility. Human Communication Research, 2008(34):347-377.

[7] Lakoff, R. The Pragmatics of Modality. In:Peranteau, P., Levi, J., Phare, G.(Eds.), Papers from the Eighth Regional Meeting. Chicago Linguistic Society, 1972:471.

[8] Prince E.F., Bosk C, \& Frader J. On Hedging in Physician-Physician Discourse[A]. Linguistics and the Profession[C], 1982.

[9] Swales, J.M., and Feak, C. Academic Writing for Graduate Students: Essential Tasks and Skills [M]. Ann Arbor, MI: University of Michigan Press, 1994.

[10] Strauss, C. Cultural standing in expression of opinion. Language in Society, 2004(33):161-164.

[11] Zeng Yuwei\& Hu Fang. Hedges in Abstracts of English Academic Papers. Shandong Foreign Language Teaching Journal, 2015 (2):4042. 